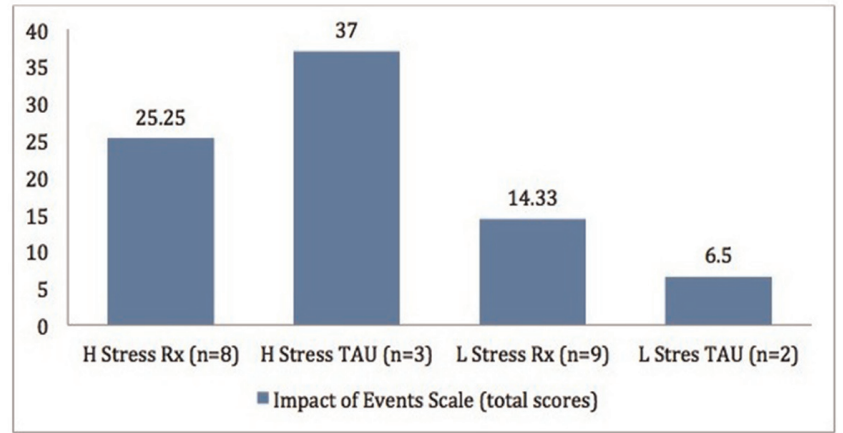

Abstract PS-137a Figure 1 Post-traumatic stress symptoms in parents at 5 months post PICU discharge in the intervention (Rx) and Treatment as usual [TAU] groups, split according to baseline parental stress score ( $\mathrm{H}=$ high stress; $\mathrm{L}=$ low stress)

Results Although not statistically significant, parents in the intervention group $(\mathrm{n}=17)$ fared better than parents in the TAU group $(\mathrm{n}=6)$ : They reported fewer PTSS [19.47 (95\% CI 11.64-26.62) vs. 25.83 (95\% CI 11.47-39.00)] and fewer were at risk for clinical disorder (18\% vs. 33\%) (Cohen's $\mathrm{d}=0.38$ and 0.34 respectively). However, there were potential implications for screening: "High stress" parents in the intervention group had lower PTSS scores than "high stress" TAU parents (d $=1.06$ ), whereas "low stress" parents in the intervention group had higher PTSS scores than "low stress" TAU parents ( $\mathrm{d}=$ 0.47) (See Figure 1).

Conclusions Pilot data indicate that a brief intervention could have a significant impact on parent PTSS following paediatic critical illness, but this needs to be evaluated in a sufficiently powered RCT.

\section{PS-137b ANESTHESIOLOGY AND INTENSIVE CARE FOR RENAL TRANSPLANTATION IN CHILDREN}

${ }^{1} Y$ Maanaoui, ${ }^{1} Y$ Rais, ${ }^{1} \mathrm{~K}$ Yaqini, ${ }^{1} \mathrm{~B}$ Hmamouchi, ${ }^{1} \mathrm{~A}$ Chlilek, ${ }^{2} \mathrm{~N}$ Mifdal, ${ }^{2} \mathrm{~K}$ Mabrouk, ${ }^{2} \mathrm{~S}$ Elkhayat, ${ }^{2} \mathrm{M}$ Zamd, ${ }^{2} \mathrm{G}$ Medkouri, ${ }^{2} \mathrm{M}$ Benghanem, ${ }^{2} \mathrm{~B}$ Ramdani. ${ }^{1}$ Paediatric Anesthesia and Intensive Care, CHU Ibn Rochd, Casablanca, Morocco; ${ }^{2}$ Nephrology Hemodialysis and Kidney Transplantation, CHU Ibn Rochd, Casablanca, Morocco

\subsection{6/archdischild-2014-307384.433}

Introduction Renal transplantation in children has special anaesthesia and postoperative intensive care, mainly for low birth weight. These features concern methods of intraoperative filling, surgical techniques and postoperative immediate resuscitation. Thus, maintaining adequate perfusion of the graft and the prevention of thrombosis of vascular anastomoses remain the main objectives of the perioperative phase. The aim of this study is to describe the procedures for anaesthesia and intensive care during surgery and immediate postoperative in paediatric renal transplantation in our unit.

Materials and methods Prospective descriptive study spread over 7 years, from January 2007 to February 2014, covering all paediatric renal transplant patients admitted to the children of our university hospital. Were collected epidemiological data of patients and grafts, duration of intubation and intensive care unit stay, haemodynamic, biological and therapeutic settings, and changing data of our patients.

Results Fourteen cases were collected with an average age of 11.32 years (range 6.5 to 16 years). Antecedents were repeated urinary tract infection (21.4\%), nephrectomy (21.4\%) and heart disease in one case. Nephropathy was the most common cause of chronic terminal renal failure (6 cases), followed by uropathies (5 cases). The dialysis modalities were peritoneal dialysis $(66.7 \%)$ and hemodialysis (33.3\%). The anaesthetic technique was by inhalation of sevoflurane in 10 cases and intravenously in 4 cases. Consisted of monitoring an invasive blood pressure (radial artery) and a central venous pressure (CVP) (jugular) in all patients. The average duration of anaesthesia was $6.64 \mathrm{~h}$. The extubation was performed after surgery in 9 cases and resuscitation in 5 cases with a mean duration of postoperative ventilation $4.6 \mathrm{~h}$. The average time of warm ischemia was $1.85 \mathrm{~h}$ and that of the $1.07 \mathrm{~h}$ of cold ischemia. Mannitol was administered in $14.3 \%$ of cases, and two cases were transfused red blood cells. Drugs administered intraoperatively were : dopamine (21.4\%), diuretics $(21.4 \%)$ and antihypertensive $(14.3 \%)$. The period of normalisation of renal function postoperatively was : day 1 (71.4\%), day $3(14.3 \%)$, day 6 and day 25 in the same proportion (7.1\%). Postoperative complications were kind of viral pneumonia in a patient, hyperglycemia in two patients, infection of the peritoneal fluid drainage in a patient and hypertension in 4 patients. The average length of intensive care unit stay was 1.28 days (range of 0.7 to 3 days). No deaths have been deplored.

Conclusion In paediatric renal transplantation, intraoperative and immediate postoperative periods emerge as the main objective of the graft infusion sufficient to prevent the occurrence of complications and ensure its survival. Although the activity of paediatric renal transplantation remains generally low in Morocco since 2007, this practice has made much progress in our country, for the survival and rehabilitation of children, once condemned.

\section{Intensive Care II}

\section{PS-138 FLUID OVERLOAD IS ASSOCIATED WITH MORTALITY IN PAEDIATRIC ACUTE RESPIRATORY DISTRESS SYNDROME (ARDS) ONLY IN THE SETTING OF ACUTE KIDNEY INJURY (AKI)}

${ }^{1} \mathrm{~A}$ Spicer, ${ }^{1} \mathrm{~V}$ Lo, ${ }^{2} \mathrm{RG}$ Khemani, ${ }^{3} \mathrm{HR}$ Flori, ${ }^{4} \mathrm{CS}$ Calfee, ${ }^{4} \mathrm{MA}$ Matthay, ${ }^{1} \mathrm{~A}$ Sapru, ${ }^{1} \mathrm{~B}$ Orwoll. ${ }^{1}$ Pediatrics, University of California San Francisco, San Francisco, USA; ${ }^{2}$ Critical Care, Children's Hospital of Los Angeles, Los Angeles, USA; ${ }^{3} \mathrm{Criti}$ al Care, Children's Hospital and Research Center Oakland, Oakland, USA; ${ }^{4}$ Medicine, University of California San Francisco, San Francisco, USA

\subsection{6/archdischild-2014-307384.434}

Background Cumulative fluid balance on day 3 is associated with mortality in paediatric ARDS (Valentine 2012, Willson 2013). Whether this association is modified by AKI is unknown. Aim To test the effect of AKI on the association between fluid and mortality in ARDS.

\begin{tabular}{lll} 
Abstract PS-138 Table 1 & Patient characteristics & \\
\hline Characteristic (Mean \pm SD) & No AKI $(n=153)$ & AKI $(n=56)$ \\
Age, Months & $86 \pm 73$ & $86 \pm 74$ \\
Male (\%) & $86(56)$ & $31(55)$ \\
P/F Ratio & $174 \pm 106$ & $156 \pm 103$ \\
PRISM 3* & $13 \pm 8$ & $21 \pm 11$ \\
Day 3 Fluid Balance $(100 \mathrm{~mL} / \mathrm{kg}){ }^{*}$ & $0.61 \pm 0.97$ & $1.1 \pm 1.59$ \\
Mortality $(\%)^{*}$ & $12(7.8)$ & $14(25)$ \\
\hline${ }^{* *} \mathrm{p}<0.01 ;$ all other $p$-values $>0.05$. & &
\end{tabular}


Abstract PS-138 Table 2 Logistic regression model adjusted for age, sex, race, PRISM 3 and inotrope use outcome: mortality predictor: day 3 fluid balance $(100 \mathrm{~mL} / \mathrm{kg})$

\begin{tabular}{lllll}
\hline & $\mathbf{n}$ & OR & $95 \% \mathrm{CI}$ & $\mathbf{p}$ \\
\hline All Patients & 209 & 1.64 & $1.14-2.37$ & 0.008 \\
Patients Without AKI & 153 & 1.35 & $0.68-2.7$ & 0.394 \\
Patients With AKI & 56 & 1.89 & $1.08-3.31$ & 0.027 \\
\hline
\end{tabular}

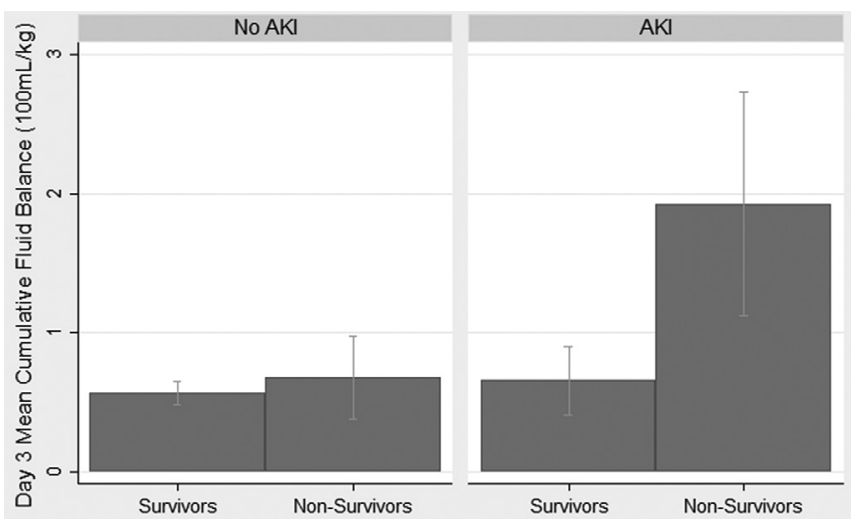

Abstract PS-138 Figure

Methods We calculated cumulative fluid balance 3 days after ARDS onset in a multi-centre cohort. AKI was defined as 'Injury' or 'Failure' by pRIFLE criteria (Akcan-Arikan 2007).

Results Patient characteristics are shown in Table 1. Mortality was higher in patients with AKI.

Fluid balance was associated with mortality independent of sex, age, race, PRISM 3 and vasopressor use; upon stratification, this association was to limited patients with AKI (Table 2 and Figure).

Conclusions Day 3 cumulative fluid balance and AKI are associated with mortality. The association with fluid balance is limited to patients with AKI. This has important implications for fluid management in ARDS patients.

\section{PS-139 NEURODEVELOPMENT WITHIN THE FIRST TWO YEARS AFTER IMPLEMENTATION OF THE EUROCONSORTIUM NEONATAL TREATMENT PROTOCOL IN CONGENITAL DIAPHRAGMATIC HERNIA PATIENTS: A MULTICENTER STUDY}

${ }^{1} \mathrm{KG}$ Snoek, ${ }^{2} \mathrm{C}$ Capolupo, ${ }^{2} \mathrm{~L}$ Aite, ${ }^{2} \mathrm{~A}$ Braguglia, ${ }^{1} \mathrm{RMH}$ Wijnen, ${ }^{2} \mathrm{~L}$ Valfrè, ${ }^{1} \mathrm{D}$ Tibboel, ${ }^{1} \mathrm{H}$ IJsselstijn. ${ }^{1}$ Department of Intensive Care and Paediatric Surgery, Erasmus MC Sophia Children's Hospital, Rotterdam, Netherlands; 'Department of Medical and Surgical Neonatology, Bambino Gesu Children's Hospital, Rome, Italy

\subsection{6/archdischild-2014-307384.435}

Background Since mortality in congenital diaphragmatic hernia $(\mathrm{CDH})$ is decreasing, morbidity such as neurodevelopmental delay receives increased attention. We evaluated neurodevelopmental outcome in high-risk $\mathrm{CDH}$ patients treated according to the Euroconsortium standardised neonatal treatment protocol (Reiss, 2010).

Methods Observational, prospective cohort study in $\mathrm{CDH}$ patients treated in two level-III university hospitals (Rotterdam (ECMO available) and Rome (no ECMO available)) between January 2009-April 2011. Sixty-three patients underwent standardised neurodevelopmental assessment at corrected age of 12

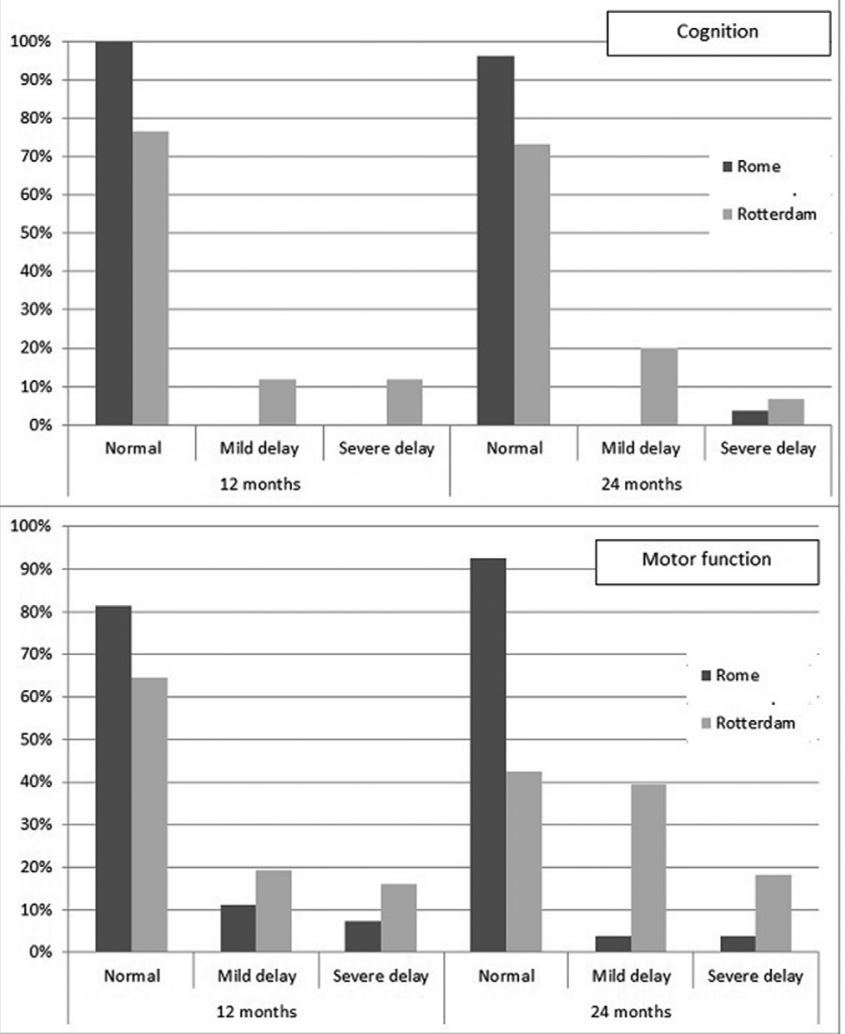

Abstract PS-139 Figure 1

and 24 months by using Bayley Scales of Infant Development (BSID)-II-NL (Rotterdam; Dutch reference data) or BSID-III (Rome; US reference data). Both centres were analysed separately due to differences in assessment instruments. Results are presented as $\mathrm{n}(\%)$, mean (SD) or median (range).

Results Mortality was $24.1 \%$ and $20.5 \%$ in Rotterdam and Rome, respectively. Baseline characteristics were for Rotterdam ( $\mathrm{n}=36) /$ Rome $(\mathrm{n}=27)$ : observed to expected lung-to-head ratio $53.8 \%(19.1) / 55.4 \%(15.8), \mathrm{p}=0.67$; SNAP-II score $(19$ $(0-40) / 7(0-25), \mathrm{p}<0.001$; chronic lung disease (i.e. oxygen dependency at day 28) in $15(41.7 \%) / 4(14.8 \%), p=0.14$; length hospital stay $21(6-387) / 27(15-82)$ days, $p=0.06$. In Rotterdam four patients $(11.1 \%)$ underwent ECMO treatment. Neurodevelopmental outcome is shown in Figure.

Conclusions At two years, the majority of $\mathrm{CDH}$ patients have normal cognition, but they are at risk for motor function delay. Future multicenter collaboration should focus on continuation of standardisation of treatment and follow-up to identify risk factors and reduce morbidity.

\section{PS-140 ¿SHOULD BE LOOK AT TIDAL VOLUMEN/WEIGHT VALUE AS A PREDICTIVE FACTOR FOR NON-INVASIVE VENTILATION FAILURE?}

${ }^{1} \mathrm{M}$ Pons-Odena, ${ }^{1} \mathrm{~L}$ Pérez Baena, ${ }^{1} \mathrm{~L}$ Rodriguez Guerinau, ${ }^{2} \mathrm{C}$ Pérez González, ${ }^{2}$ A Medina Villanueva, ${ }^{3}$ J López González, ${ }^{4} D$ Palanca Arias, ${ }^{3}$ J López-Herce, ${ }^{4} \mathrm{P}$ Garcia Iñiguez. 'PICU, Hospital Sant Joan de Déu, Barcelona, Spain: ${ }^{2} P I C U$, Hospital Universitario Central Asturias, Oviedo, Spain; ${ }^{3} P I C U$, Hospital Universitario Gregorio Marañón, Madrid, Spain; ${ }^{4}$ PICU, Hospital Universitario Miguel Servet, Zaragoza, Spain

\subsection{6/archdischild-2014-307384.436}

Background and aims Expected normal tidal volume values have not been described in children treated on NIV yet. Describe 